\title{
Diffusion MRI data, sulcal anatomy, and tractography for eight species from the Primate Brain Bank
}

\author{
Katherine L. Bryant ${ }^{1}$ - Dirk Jan Ardesch ${ }^{2}$ (1) - Lea Roumazeilles ${ }^{3}$ - Lianne H. Scholtens ${ }^{2}$ - Alexandre A. Khrapitchev ${ }^{4}$. \\ Benjamin C. Tendler ${ }^{1}$. Wenchuan $\mathrm{Wu}^{1} \cdot$ Karla L. Miller ${ }^{1}$. Jerome Sallet ${ }^{3,5} \cdot$ Martijn P. van den Heuvel $^{2,6}$. \\ Rogier B. Mars ${ }^{1,7}$
}

Received: 27 January 2021 / Accepted: 26 March 2021 / Published online: 15 July 2021

(c) The Author(s) 2021

\begin{abstract}
Large-scale comparative neuroscience requires data from many species and, ideally, at multiple levels of description. Here, we contribute to this endeavor by presenting diffusion and structural MRI data from eight primate species that have not or rarely been described in the literature. The selected samples from the Primate Brain Bank cover a prosimian, New and Old World monkeys, and a great ape. We present preliminary labelling of the cortical sulci and tractography of the optic radiation, dorsal part of the cingulum bundle, and dorsal parietal-frontal and ventral temporal-frontal longitudinal white matter tracts. Both dorsal and ventral association fiber systems could be observed in all samples, with the dorsal tracts occupying much less relative volume in the prosimian than in other species. We discuss the results in the context of known primate specializations and present hypotheses for further research. All data and results presented here are available online as a resource for the scientific community.
\end{abstract}

Keywords Comparative $\cdot$ Connectivity $\cdot$ Cortex $\cdot$ Anthropoid elaboration $\cdot$ Optic radiation $\cdot$ Cingulum bundle

\section{Introduction}

The comparative method is one of the primary ways to gain insight into brain evolution. In the study of humans and other primates, this paradigm has been very successful in elucidating the evolution of the size of the brain and some its constituent parts [e.g., Miller et al. (2019); Smaers and Vanier (2019)]. Although these methods are able to

Katherine L. Bryant and Dirk Jan Ardesch contributed equally.

Rogier B. Mars

rogier.mars@ndcn.ox.ac.uk

1 Wellcome Centre for Integrative Neuroimaging, Centre for fMRI of the Brain (FMRIB), Nuffield Department of Clinical Neurosciences, John Radcliffe Hospital, University of Oxford, Headington, Oxford OX9 3DU, UK

2 Department of Complex Trait Genetics, Centre for Neurogenomics and Cognitive Research, Amsterdam Neuroscience, Vrije Universiteit Amsterdam, Amsterdam, The Netherlands

3 Wellcome Centre for Integrative Neuroimaging, Department of Experimental Psychology, University of Oxford, Oxford, UK identify specializations in different branches of the primate tree, including instances of mosaic evolution (Barton and Harvey 2000; Smaers and Soligo 2013), a poverty of data in this field has also meant that few datasets are available and definition of constituent parts is often difficult to determine, giving rise to strong controversies in the field. A prominent example of such a debate is whether the size of human prefrontal cortex follows trends expected based on other primates (Barton and Venditti 2013; Passingham and Smaers 2014). Moreover, the focus on size or relative

4 Department of Oncology, University of Oxford, Cancer Research UK and Medical Research Council Oxford Institute for Radiation Oncology, Oxford, UK

5 Univ Lyon, Université Lyon 1, Inserm, Stem Cell and Brain Research Institute U1208, 69500 Bron, France

6 Department of Clinical Genetics, Amsterdam Neuroscience, Amsterdam UMC, Vrije Universiteit Amsterdam, Amsterdam, The Netherlands

7 Donders Institute for Brain, Cognition and Behaviour, Radboud University Nijmegen, Nijmegen, The Netherlands 
size of the brain or its parts obscures the fact that many other changes can have occurred across different lineages, including changes in the connections of the brain and in the number of areas (Krubitzer and Kaas 2005; Mars et al. 2017). However, the labor intensive and destructive nature of many of the traditional techniques to study such changes makes them unfeasible for large-scale comparisons.

The rise of neuroimaging as a tool in comparative neuroscience over the last decade has the potential to address these problems (Mars et al. 2014). Neuroimaging techniques such as magnetic resonance imaging (MRI) have the advantage that the brains can be scanned in whole, potentially using different sequences, without destroying or damaging the tissue (Thiebaut De Schotten et al. 2019). This means that tissue that has been scanned remains available for further analyses, whether using neuroimaging or more traditional techniques. The digital nature of neuroimaging data makes data sharing easier, facilitating direct comparisons across various datasets. As neuroimaging and traditional techniques are often the expertise of different labs, this type of data sharing is crucial to reach a full understanding of brain diversity. Neuroimaging thus has potential beyond producing new data; it allows a fusion of data types describing brain organization at different levels of resolution (McKavanagh et al. 2019). This, in turn, enhances understanding of brain diversity at multiple scales.

Diffusion MRI in particular has recently proven a popular tool in comparative neuroimaging. Tractography algorithms allow one to use these data to reconstruct the major fiber bundles of the brain, which are an excellent starting point for quantitative comparisons of brain organization. Pioneering work on comparative tractography identified the arcuate fascicle, one of the major tracts connecting areas involved in language processing in the human brain, as expanded in the human as compared to the macaque and chimpanzee brain (Rilling et al. 2008). Subsequent work has investigated this result in the light of other changes between these brains
(Ardesch et al. 2019; Eichert et al. 2019) and embarked on systematic comparisons of tracts between human and nonhuman brains (Hecht et al. 2015; Mars et al. 2016; Takemura et al. 2017; Bryant et al. 2019). The creation of whole-brain tract libraries for various species (Thiebaut de Schotten et al. 2012; Bryant et al. 2020; Warrington et al. 2020), advances in data processing ( $\mathrm{Li}$ et al. 2013; Mars et al. 2019), and integration with other modalities (Eichert et al. 2020) increasingly open up new avenues for novel comparisons of brain organization.

To facilitate this endeavor, we here present high-quality structural and diffusion MRI data from eight primate species, distributed across the different branches of the primate tree. All samples were acquired from the Primate Brain Bank (PBB), a non-profit initiative at the Netherlands Institute for Neuroscience that collects brain samples from deceased primates from a number of zoos in the Netherlands (Kaas and van Eden 2011). We also present labelling of sulcal anatomy based on reconstructions of the brains' cortical surfaces and tractography of some of the major white matter fiber bundles using standardized protocols. All data and results are available online to the scientific community.

\section{Materials and methods}

\section{Samples and scanning}

We acquired eight brains from the Primate Brain Bank (PBB) — one prosimian primate (bushbaby), four New World monkeys (night monkey, woolly monkey, white-faced saki, and tufted capuchin), two Old World monkeys (black-andwhite colobus and grey-cheeked mangabey), and one great ape (chimpanzee). Demographics are listed in Table 1. All samples were returned to the PBB after scanning and remain available for future research. In addition, we used data from one rhesus macaque (Macaca mulatta, male, 4.03 years of
Table 1 Demographic information of the scanned PBB samples

\begin{tabular}{lllll}
\hline Latin name & Common name & Sex & Age (years) & PBB identifier \\
\hline $\begin{array}{l}\text { Prosimians } \\
\text { Galago senegalensis }\end{array}$ & Senegal bushbaby & M & 20.9 & PB0123 \\
$\begin{array}{l}\text { New world monkeys } \\
\text { Pithecia pithecia }\end{array}$ & White-faced saki & M & 4 & \\
Lagothrix lagotricha & Woolly monkey & F & 8 & PB0720 \\
Sapajus apella & Tufted capuchin & M & 22 & PB0630 \\
Aotus lemurinus & Night monkey & M & 15.3 & PB0737 \\
Old world monkeys & & & & PB0425 \\
$\begin{array}{l}\text { Colobus guereza } \\
\text { Lophocebus albigena }\end{array}$ & Black-and-white colobus & M & 23.7 & PB0113 \\
Great apes & & F & 27 & PB0714 \\
Pan troglodytes & Chimpanzee & & & PB1301 \\
\hline
\end{tabular}


age at death) from a previous study (Folloni et al. 2019) for comparison with this most commonly studied primate. Phylogenetic relationships between all species in this study and the human are displayed in Fig. 1.

Prosimian and monkey data were acquired locally on a $7 \mathrm{~T}$ magnet with an Agilent DirectDrive console (Agilent Technologies, Santa Clara, CA, USA) using a 2D diffusionweighted spin-echo protocol with single line readout (DWSEMS, TE/TR: $25 \mathrm{~ms} / 10 \mathrm{~s}$; Matrix $=128 \times 128$; number of slices: 128 ; Resolution: $0.3 \times 0.3 \times 0.3 \mathrm{~mm}$ for the galago, $0.4 \times 0.4 \times 0.4 \mathrm{~mm}$ for the night monkey, $0.5 \times 0.5 \times 0.5 \mathrm{~mm}$ for the saki monkey, $0.6 \times 0.6 \times 0.6 \mathrm{~mm}$ for the woolly, capuchin, colobus, and mangabey; diffusion data were acquired over the course of $\sim 52.5 \mathrm{~h}$ ). 16 non-diffusion-weighted $\left(b=0 \mathrm{~s} / \mathrm{mm}^{2}\right)$ and 128 diffusion-weighted $\left(b=4000 \mathrm{~s} / \mathrm{mm}^{2}\right)$ volumes were acquired with diffusion directions distributed over the whole sphere. The brains were soaked in PBS before scanning and placed in fluorinert during the scan.

Great ape data were acquired on a $7 \mathrm{~T}$ whole-body scanner with an 28 channel knee coil (QED) using a 3D diffusion-weighted steady-state free precession (McNab et al. 2009) protocol (DW-SSFP, TE/TR: $18 \mathrm{~ms} / 22 \mathrm{~ms}$; flip angle: $50^{\circ}$; resolution: $0.6 \times 0.6 \mathrm{~mm}^{2}$; number of slices:
176; slice thickness: $0.6 \mathrm{~mm}$; EPI factor: 1; diffusion data were acquired over the course of $\sim 50 \mathrm{~h}$ ). 12 non-diffusionweighted $\left(q\right.$-value $\left.=20 \mathrm{~cm}^{-1}\right)$ and 240 diffusion-weighted $\left(q\right.$-value $\left.=300 \mathrm{~cm}^{-1}\right)$ volumes were acquired with diffusion directions distributed over the whole sphere. Accurate modelling of DW-SSFP data requires the estimation of DW-SSFP dependencies (T1, T2 and B1) (Buxton 1993). To account for this, $\mathrm{T}_{1}$-weighted data were obtained using a turbo inversion recovery (TIR, TIs: 30, 60, 120, 240, 480 and $940 \mathrm{~ms}$; TE/TR: $17 \mathrm{~ms} / 1020 \mathrm{~ms}$; resolution: $0.8 \times 0.8$ $\mathrm{mm}^{2}$; number of slices: 112 ; slice thickness: $0.8 \mathrm{~mm}$; TIR data acquired over the course of $\sim 2 \mathrm{~h}$ ), $\mathrm{T}_{2}$-weighted data were obtained using a turbo spin-echo (TSE, TEs: 8.7, 17, 26, 35, 52 and $70 \mathrm{~ms}$; TR: $1000 \mathrm{~ms}$; resolution: $0.8 \times 0.8$ $\mathrm{mm}^{2}$; number of slices: 112 ; slice thickness: $0.8 \mathrm{~mm}$; TSE data acquired over the course of $\sim 2 \mathrm{~h}$ ) and $\mathrm{B}_{1}$ maps were estimated using an actual flip angle imaging (Yarnykh 2007) sequence (AFI; TE: $2.6 \mathrm{~ms}$; TRs: 7.7 and $21 \mathrm{~ms}$; resolution: $3 \times 3 \mathrm{~mm}^{2}$; number of slices: 32 ; slice thickness: $3 \mathrm{~mm}$; AFI data acquired in $\sim 43 \mathrm{~s}$ ).

MGE data for surface reconstruction for the prosimian and monkey samples from the PBB were also acquired on a $7 \mathrm{~T}$ magnet with an Agilent
Fig. 1 Phylogenetic relationships between the species in this study. Numbers indicate median divergence times (in millions of years) based on genomic data (Hedges et al. 2015)

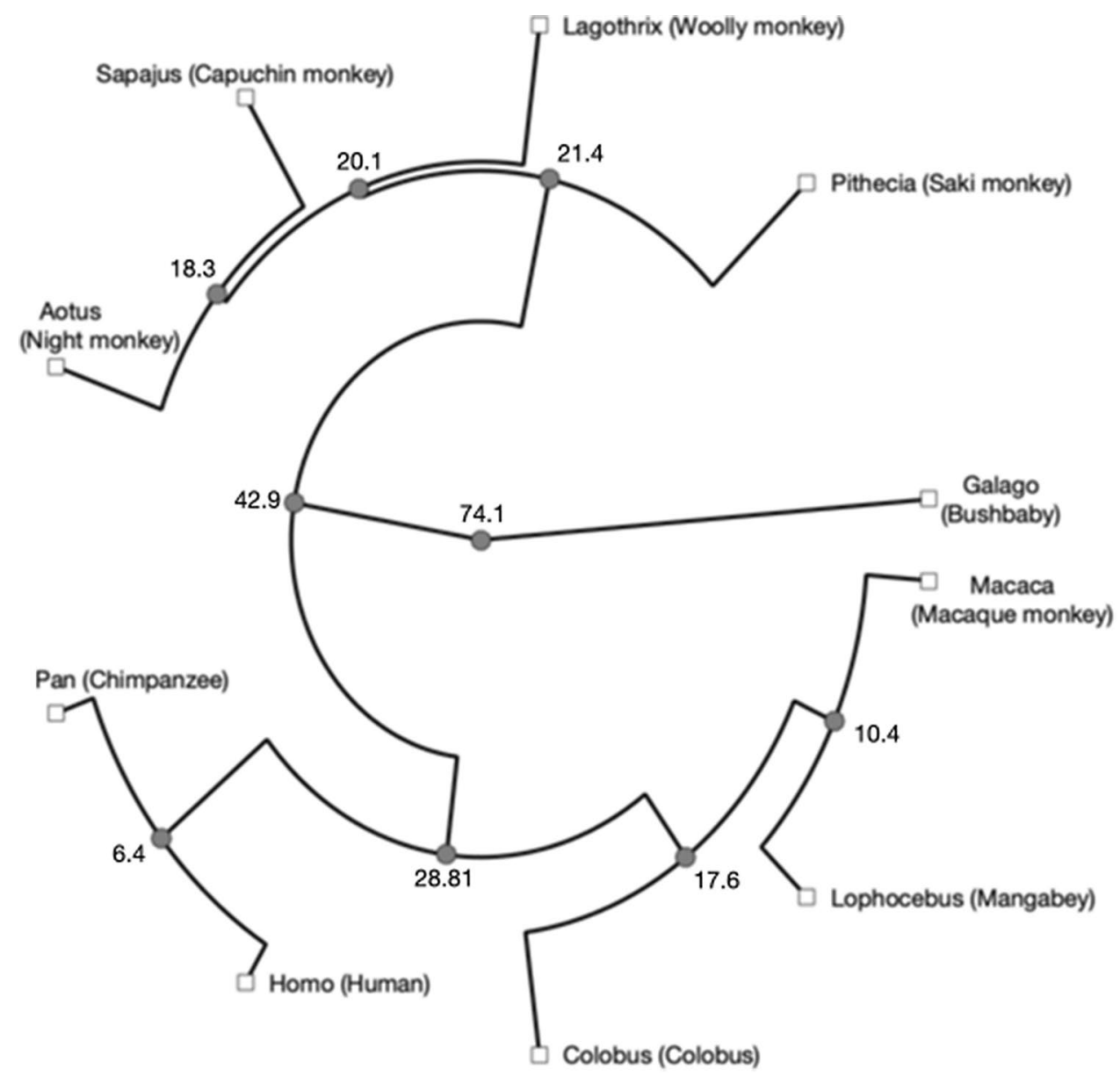


DirectDrive console (Agilent Technologies, Santa Clara, CA, USA) using a 3D multi-gradient-echo sequence (MGE3D, TE1/8TE/TR $=7.5 \mathrm{~ms} / 11 \mathrm{~ms} / 98 \mathrm{~ms}$; resolution $=0.15 \times 0.15 \times 0.15 \mathrm{~mm}($ galago $), 0.2 \times 0.2 \times 0.2 \mathrm{~mm}$ (night monkey), $0.25 \times 0.25 \times 0.25 \mathrm{~mm}$ (saki monkey), $0.3 \times 0.3 \times 0.3 \mathrm{~mm}$ (other samples); data were acquired over $1 \mathrm{~h})$.

Structural scans for the great ape were acquired on a $7 \mathrm{~T}$ whole-body scanner with a 28 channel knee coil (QED) using a 3D true fast imaging with steady state free precession protocol (TRUFI, TE/TR: $5.06 \mathrm{~ms} / 10.12 \mathrm{~ms}$; flip angle: $39^{\circ}$; resolution $=0.4 \times 0.4 \mathrm{~mm}^{2}$; number of slices: 256 ; slice thickness: $0.4 \mathrm{~mm}$; TRUFI data acquired over the course of $22 \mathrm{~min}$ ). 4 TRUFI datasets were acquired (phase increment: $0^{\circ}, 90^{\circ}, 180^{\circ}$, and $270^{\circ}$ ) and averaged via root sum of squares.

\section{Diffusion MRI data preprocessing}

All prosimian and monkey datasets were converted to NIFTI format, reoriented to approximate AC/PC orientation with the origin set at the middle of the anterior commissure, and preprocessed using tools from FSL (www.fmrib.ox.ac.uk/ fsl). These steps are implemented in the 'phoenix' module of the MR Comparative Anatomy Toolbox (Mr Cat; www. neuroecologylab.org). We used BedpostX to fit a crossing fiber model to each dataset (Behrens et al. 2007), allowing up to three fiber orientations per voxel. This tool produces voxel-wise posterior distributions of fiber orientations that are subsequently used for probabilistic tractography.

For the great ape DW-SSFP data, prior to analysis a Gibbs-ringing correction (Kellner et al. 2016) was performed on both the diffusion-weighted and non-diffusionweighted datasets. To account for the DW-SSFP dependencies $\left(T_{1}, T_{2}\right.$ and $\left.B_{1}\right), T_{1}$ and $T_{2}$ maps were estimated from the TIR and TSE datasets assuming mono-exponential signal evolution (the Gibbs correction was also performed on the TIR and TSE data). A $B_{1}$ map was generated from the AFI data following the methodology described in Yarnykh (2007). All co-registrations between and within modalities were performed using FSL FLIRT using a 6 degrees-of-freedom (rotation and translation) transformation. These datasets were subsequently fit using custom diffusion tensor and ball and two stick models which incorporates the full Buxton DW-SSFP signal equations (Buxton 1993) using cuDIMOT (Hernandez-Fernandez et al. 2019).

\section{Surface reconstruction}

Cortical surfaces were reconstructed from the structural scans using FreeSurfer v6.0 (Fischl 2012). Processing steps included tissue segmentation of grey and white matter, identification of subcortical structures, and surface reconstruction. To obtain successful reconstructions of the non-human primate brains, the FreeSurfer pipeline was complemented with tools from FSL v6.0.1 (Jenkinson et al. 2012), ANTs (Avants et al. 2011), and MATLAB (2017a, The Mathworks, Inc., Natick, Massachussets, United States).

A brain mask was used to separate brain tissue from any other structures present in the scans such as fiducial markers. Bias field correction was then applied to the images with ANTs. Voxel intensities from the original MGE and TRUFI images were inverted to obtain a T1-like contrast with low voxel intensities in gray matter voxels and high intensities in white matter voxels. For the mangabey, an average b0 volume was used as the original image instead. Next, tissue segmentation was performed using FreeSurfer. Manual corrections were made where necessary to correct segmentations of white matter, cerebellum, and subcortical structures. FreeSurfer's default Talairach registration step was complemented by a step-by-step registration process, starting with a registration from the subject at hand to a macaque template brain (Seidlitz et al. 2018), followed by a registration from the macaque template to a chimpanzee template brain (based on data from chimpanzeebrain.org), and from the chimpanzee template to human Talairach space. The chimpanzee brain was first registered to the chimpanzee template brain and then to human Talairach space, skipping the macaque template step. This step-by-step registration process ensured that major brain structures such as the cerebellum and thalamus were correctly segmented in the smaller brains. Next, surface reconstruction was started on the high-resolution volumes. The pial surface was refined by alignment to a downsampled version of the original volumes using the high-resolution scripts from the HCP FreeSurfer pipeline (https://github.com/Washington-University/HCPpipelines/ tree/master/FreeSurfer/custom). Tissue segmentations and surface reconstructions were visually checked for accuracy and consistency across datasets. It should be noted that the reliance on manual interference does produce some artifacts, especially in the territory underneath the corpus callosum. This subcortical area, however, is usually masked out when working with cortical surface data.

Surfaces were converted to GIFTI format and a midthickness surface was created by averaging the pial and white matter border surfaces. For display purposes, the midthickness surface was smoothed (strength $0.5,15$ iterations) using the Workbench Command tools of Connectome Workbench (Marcus et al. 2011).

\section{Tractography}

Tractography recipes were defined using the conventions of XTRACT, a recently released tool to standardize tractography across species (Warrington et al. 2020). Each tract was defined through a seed and a target or waypoint mask, a set 
of exclusion masks, and potentially a stop mask. All recipes were drawn with respect to homologous sulci and gyri or as similar a location as possible. All recipes are available online (see "Data availability" statement). These tracts give a good balance between association and non-association fibers and fibers crossing through areas with and without crossing fibers.

\section{Optic radiation (OR)}

The optic radiation consists of fibers from the lateral geniculate nucleus (LGN) of the thalamus to the primary visual cortex. It was seeded in the LGN and the target mask consisted of a coronal plane just anterior to the lunate sulcus. Exclusion masks consisted of an axial block of the brainstem, a coronal block of fibers directly posterior to the LGN to select fibers that curl around dorsally, and a coronal plane anterior to the seed to prevent leaking into longitudinal fibers.

\section{Cingulum bundle, dorsal segment (CBD)}

The dorsal segment of the cingulum bundle was seeded just at the front of the posterior third of the corpus callosum and had a target at the start of the genu of the corpus callosum. A mid-sagittal exclusion mask prevented contralateral tracts and a coronal mask through the territory of the superior longitudinal fasciculi at the level of the midpoint of the corpus callosum prevented leakage into this system. Finally, an axial exclusion mask below the corpus callosum ensured that we only picked up tracts belonging to the dorsal segment of the cingulum bundle. Tractography was run with the seed and target masks and again with the roles of the two masks reversed and the results were averaged.

\section{Superior longitudinal fascicle complex (SLFc)}

The three branches of the superior longitudinal fasciculus are reconstructed together using an extension of the approach taken by Thiebaut de Schotten et al. (2011). In each case, a coronal plane in the region of the central sulcus (if present) is used as a seed along with two target masks. Frontally, a target mask through the territory of the SLFc was placed at the level of the anterior commissure. Posteriorly, a large coronal target mask in the parietal cortex, was placed immediately posterior to the margin of the cingulate gyrus, if present. Exclusion masks were placed in the midsagittal plane, axially to exclude tracts running ventrally of parietal-frontal cortex, and through the cingulum bundle at the level of each seed and target mask.

\section{Longitudinal fronto-temporal tract (LFT)}

A ventral longitudinal tract, often termed inferior frontooccipital fascicle (IFOF) or extreme capsule fiber complex, has been identified using tractography in the human (Forkel et al. 2014), and more recently in great apes and monkeys (Mars et al. 2016; Roumazeilles et al. 2020). It courses from ventral frontal cortex, through the extreme capsule, running medially through the temporal cortex, reaching the occipital lobe. In larger primates the IFOF's location can be separated from the temporal middle and inferior longitudinal fascicles, but this becomes difficult in species where the temporal cortex is less gyrified. Therefore, we here placed a seed in the extreme capsule connecting temporal and frontal cortex and used an entire coronal section of temporal white matter at the level of the posterior commissure as target mask. A coronal exclusion mask encompassing the entire white matter except for the seed prevented spurious anterior-posterior tracts and a sagittal exclusion masks prevented leakage into the other hemisphere. Tractography was run with the seed and target masks and again with the roles of the two masks reversed and the results were averaged.

Tractography was performed using probtrackx 2 , using the default parameters except for the step length $(0.15 \mathrm{~mm}$ for all samples apart from 0.1 for the galago). 1000 samples were seeded in each seed voxel. The resulting tractograms were normalized with respect to the total number of valid streamlines generated to allow comparison between species.

\section{Results}

We collected high-quality diffusion and structural MRI data from eight primate species that are not or little described in the comparative neuroimaging literature, as well as presenting data from the well-studied rhesus macaque. Samples were selected for their high quality and the absence of many large cuts due to the brain extraction process. Nevertheless, some cuts were present and evident in the imaging data around the occipitotemporal cortex in the night monkey, capuchin monkey, woolly monkey, and colobus monkey (Fig. 2). In addition, the saki monkey does not have a cerebellum attached anymore.

\section{Sulcal anatomy}

Figure 2 shows a lateral view of a midthickness surface for the scanned prosimian and monkey samples and the macaque F99 surface (Van Essen et al. 2012) for comparison. We did not label the chimpanzee, as this great ape brain's morphology is very different from the (pro)simian 
samples and a comprehensive atlas was recently published (Falk et al. 2018). Sulci were labelled predominantly using terms from the rhesus macaque or, if those were not present, the description of Connolly (1950) as a guide. As described before, the galago cortex shows very little gyrification, with the lateral and intra-parietal sulci as the only prominent sulci on the lateral surface and only dimples present in other locations. The night monkey shows only moderately more gyrification, with a quite extended lateral sulcus and a temporal longitudinal sulcus.

In the frontal cortex, most monkeys show a clear principal sulcus [previously termed the sulcus rectus, Connolly (1950)] and, except for the saki and night monkeys, a clear arcuate sulcus. The relative location of the arcuate sulcus and the presence of a spur at the back of it are variable, with some of the larger brains showing a clear posterior superior frontal dimple. On the medial wall, a cingulate sulcus running above the corpus callosum is evident in all species. However, the marginal sulcus is not present in the galago and only to a very limited extent in the night monkey.

The complexity of the occipital-temporal area also differs substantially across species, ranging from no gyrification in the galago, little in the saki monkey, to clear lunate and inferior occipital sulci in the capuchin, mangabey, and macaque. The intra-parietal sulcus is generally present, but its relationship with the posterior ends of the lateral and superior temporal sulci is variable. The most complex posterior gyrification can be seen in the woolly monkey, with distinct segments where the intraparietal sulcus is in Old World monkeys.

\section{Galago senegalensis (Bushbaby)}
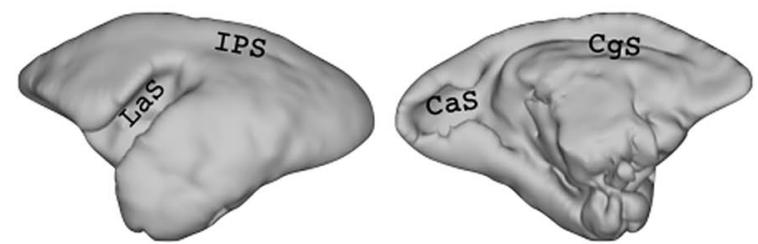

Lagothrix lagotricha (Woolly monkey)
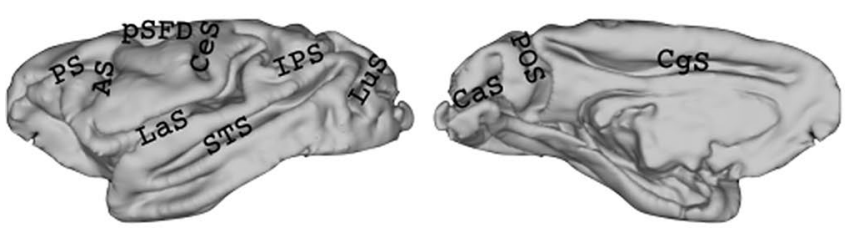

Aotus lemurinus (Night monkey)
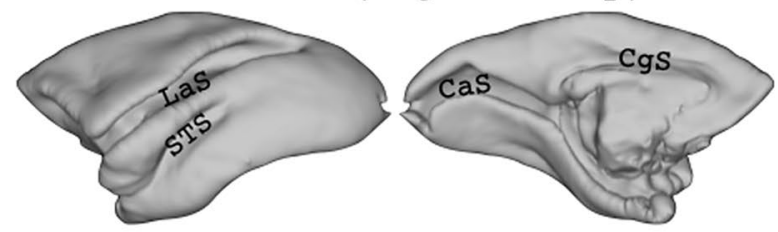

Lophocebus albigena (Mangabey)
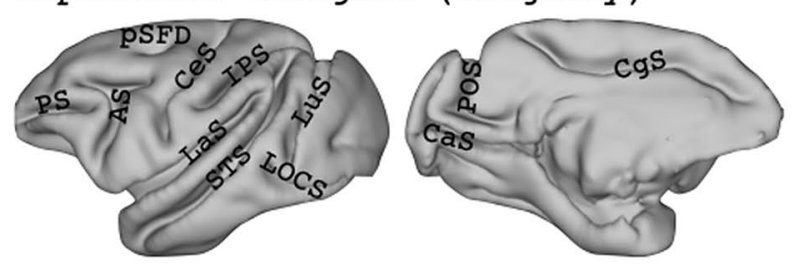

Fig. 2 Preliminary sulcal labelling on the lateral surface of the cortical hemisphere of the prosimian and monkey species in this study. All surfaces were reconstructed from the scanned sample except for the macaque, for which we used the F99 template brain (Van Essen et al. 2012). Left hemisphere lateral sulcus on the left, medial surface on the right. $A S$ arcuate sulcus, $\mathrm{CaS}$ calcarine sulcus, $\mathrm{CeS}$ central sulcus,
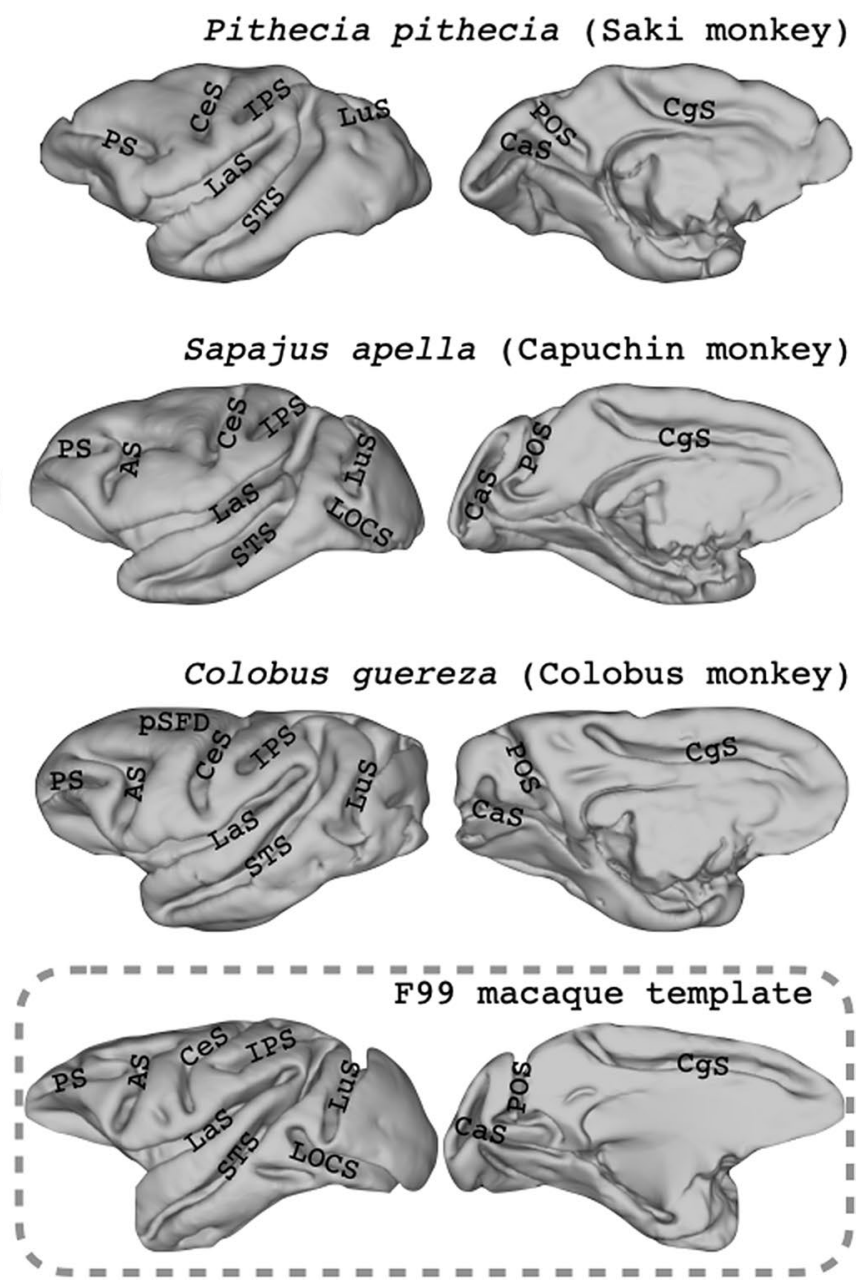

$C g S$ cingulate sulcus, $L O C S$ lateral occipital sulcus, IPS intra-parietal sulcus, $L a S$ lateral sulcus, $L u S$ lunate sulcus, $P O S$ parietal-occipital sulcus, $P S$ principal sulcus, $p S F D$ posterior superior frontal dimple, $S T S$ superior temporal sulcus. Note that brains are not displayed to scale 


\section{White matter anatomy and tractography}

We were able to obtain high-quality diffusion MRI data in all species. The pattern of orientation of the principal diffusion direction allows us to identify some of the prominent landmarks of the primate white matter (Fig. 3). Between hemispheres, the corpus callosum is the most prominent bundle, but the anterior commissure was also very present and could be used as the origin of the image. The posterior commissure was sometimes more difficult to identify, but can be used to orient the images to approximate AC/PC orientation. Prominent projections fibers, especially the upcoming fibers of the corticospinal tract and internal capsule were clearly present. In addition, anterior-posterior longitudinal fibers were clearly visible, even in areas of crossing fibers where they were not evident in the primary diffusion direction.
As a first reconstructed tract, we identified the optic radiation, a projection fiber tract carrying fibers from the LGN toward the primary visual cortex. This tract was identified in all species (Fig. 4). The same was true for the dorsal part of the cingulum, a longitudinal fiber bundle running just above and along the length of the corpus callosum. However, while this fiber tract is generally a separated bundle running through the cingulate gyrus, in the smaller brains, including the galago and night monkey, it consisted of a white matter sheet that was continuous with parts of the superior longitudinal fibers (Fig. 3).

The superior longitudinal fascicles (SLF) run dorsally between the parietal and frontal cortex. These fibers run through territory with multiple crossing fibers and as a result can be difficult to reconstruct (Behrens et al. 2007; Mars et al. 2019). Indeed, parts of the SLF can be seen in the secondary fiber directions of all species, including the galago.

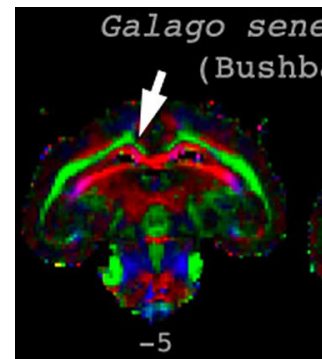

\section{galensis}

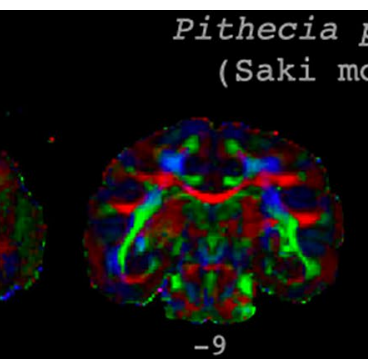

pithecia

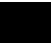

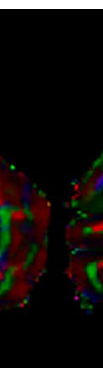

Lagothrix lagotricha

0
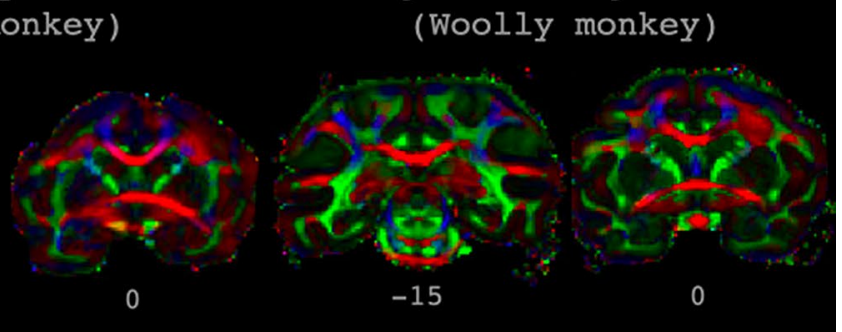

Sepajus apella

(Capuchin monkey)

Aotus lemurinus

(Night monkey)

Colobus guereza

(Colobus monkey)
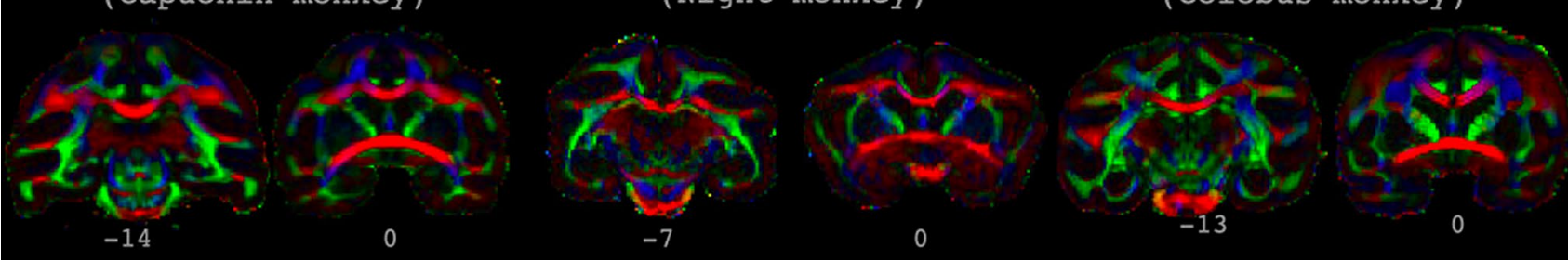

Lophocebus albigena

Pan troglodytes

(Mangabey)

(Chimpanzee)
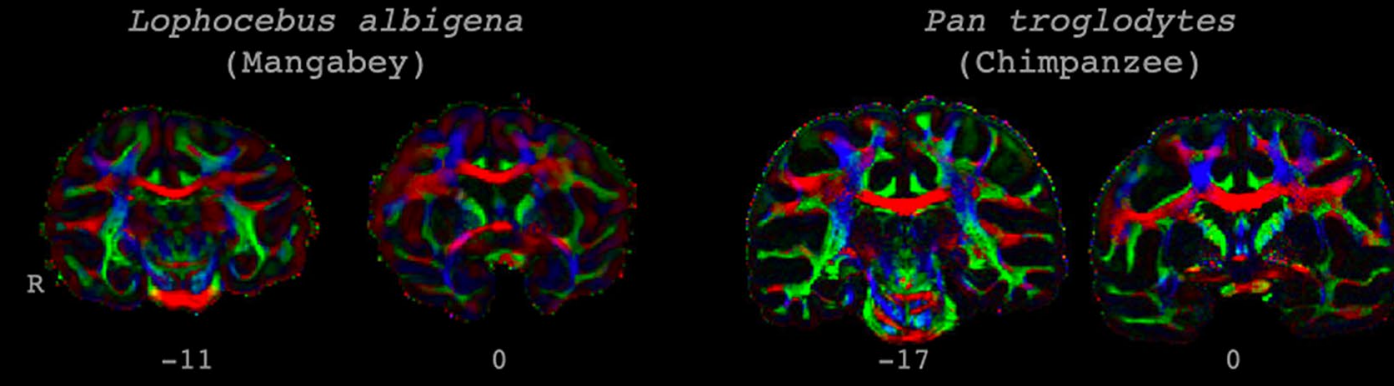

Fig. 3 Principal diffusion direction maps. Images show coronal sections of the samples in this study at the level of the anterior commissure $(y=0)$ and the level approximately $25 \%$ from the posterior edge of the corpus callosum. As can be seen, the temporal white matter of bushbaby consists of a single sheet, rather than the more extensive pattern seen in more encephalized species, including the woolly monkey. Similarly, in the bushbaby and night monkey, the cingulum bundle consists of a white matter sheet that is difficult to distinguish from the superior longitudinal fascicle complex, as indicated for the bushbaby by the white arrow. Colors indicate principal directions, red left-right, green anterior-posterior, blue dorsal-ventral. Note that brains are not displayed to scale 
We here sought to establish whether we could reconstruct longitudinal tracts between parietal and frontal cortex in all species. To increase the sensitivity, we aimed to reconstruct the entirety of the SLFs as a single 'complex', rather than distinguishing the individual subbranches as is often done in more gyrified species, including the human and macaque (Schmahmann and Pandya 2006; Thiebaut de Schotten et al. 2011). Figure 4 shows that we could identify such tracts in all species.

In the human brain, a prominent fiber bundle termed the inferior fronto-occipital fascicle (IFOF) travels through the extreme capsule, connecting parts of occipital and temporal cortex with the frontal cortex. In more gyrified species, the IFOF can be distinguished from the middle and inferior longitudinal fascicles (MdLF, ILF) running in the gyri of the temporal lobe. However, in the prosimian galago the temporal white matter consists primarily of a single sheet making it difficult to distinguish sub-tracts. We therefore reconstructed what we termed a longitudinal frontotemporal tract (LFT) that should pick up the IFOF, but might include sections of MdLF, ILF, and uncinate fascicle, by selecting the entire coronal section of the temporal white matter in the target mask. We did keep the constraint of fibers having to pass through the extreme capsule to be included in the tractogram; this was done to make it more likely we isolated fibers belonging to the IFOF. We observed such a fiber running between frontal and occipitotemporal cortex in all species studied here, including the prosimian galago. In the galago, the bulk of the tractogram runs in the middle part of the temporal

\section{Galago senegalensis (Bushbaby)}
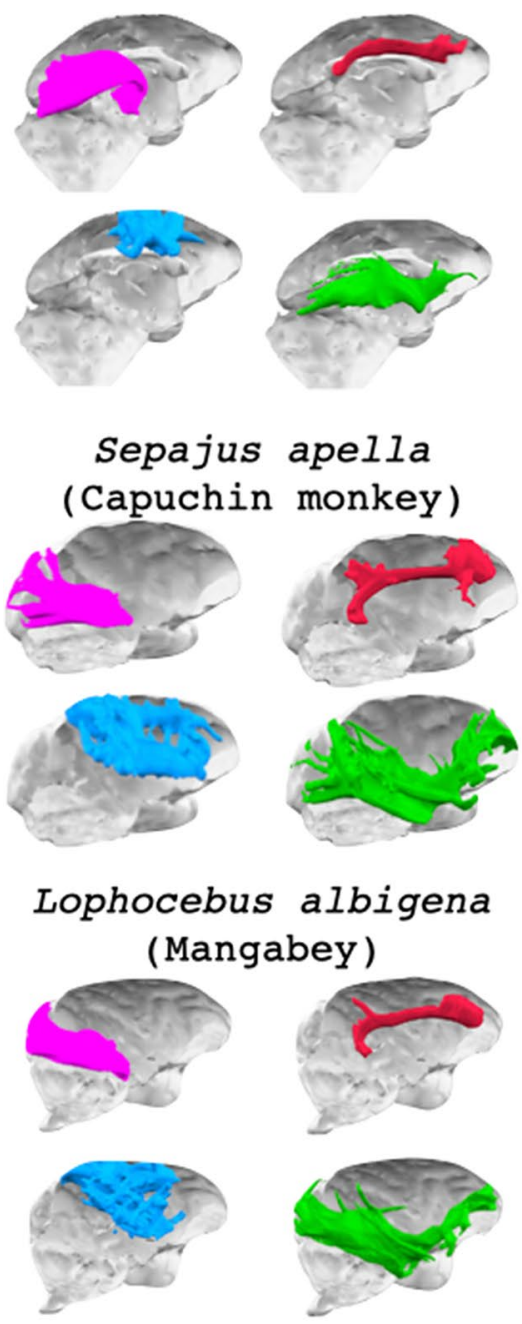

\section{Pithecia pithecia (Saki monkey)}
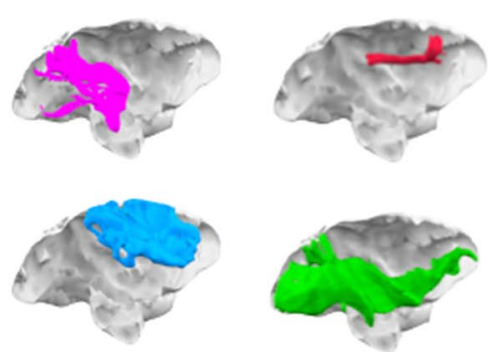

\section{Aotus lemurinus \\ (Night monkey)}

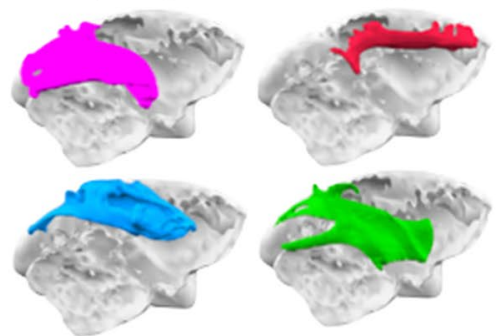

Pan troglodytes

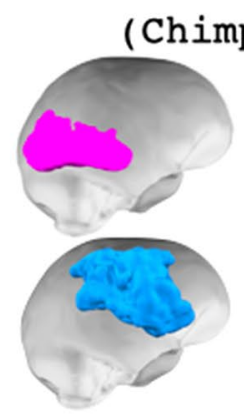

\section{Lagothrix lagotricha (Woolly monkey)}
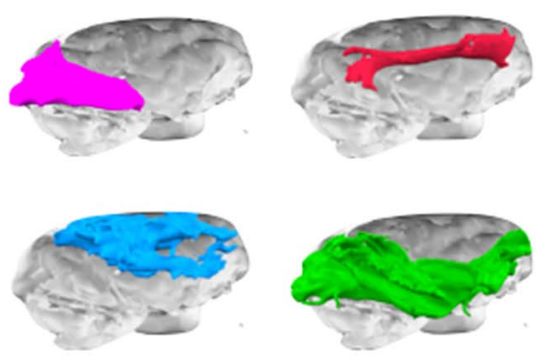

\section{Colobus guereza (Colobus monkey)}
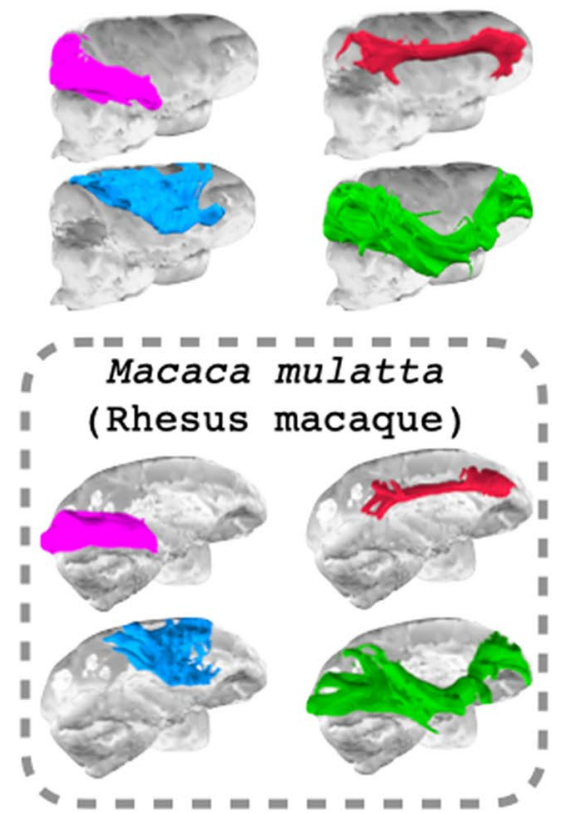

Fig. 4 Course of reconstructed white matter tracts. 3d representations of the course of cingulum bundle (red), optic radiation (green), longitudinal fronto-temporal tract (blue), and SLF complex (purple) through the brains of all studied species 
white matter sheet, which is consistent with the location of the IFOF in other species.

The longitudinal frontotemporal tract and the SLF complex are association fibers, connecting parts of association cortex that have preferentially expanded in some parts of the primate lineage (Chaplin et al. 2013). As such, one might expect these fibers to occupy more of the cortical white matter in anthropoid primates than in prosimians. To account for the size of the brain itself, we compared the volume of the association tracts with those of the non-association tracts (Fig. 5). This showed, albeit qualitatively, that the SLF complex of parietal-frontal fibers was least elaborate in the galago compared to all other primates. The same relationship was not observed for the fronto-temporal fibers.

\section{Discussion}

Gaining a full understanding of the similarities and differences across different species' brains requires multiple types of data that are available in multiple species, allowing comparisons across different scales within a species followed by comparisons between species using the same data types. We have termed these comparisons vertical translations and horizontal translations (Mars et al. 2021), respectively, and demonstrated that both are necessary to gain a full understanding of brain diversity (Eichert et al. 2020). Here, we present data on sulcal morphology and white matter anatomy from eight primate species that are not or hardly described in the literature. The data are freely available to allow quantitative comparisons across species. Importantly, the brain samples are still available at the Primate Brain Bank, opening the door for acquisition of more, including potentially more destructive, data types from these brains to increase the number of possible comparisons.

Sulcal labelling in the samples presented here is based on similarity in morphology and location of the sulci. Mostly, we based our terminology on labels commonly used in the macaque, supplemented by descriptions by Connolly (1950). Overall, the major sulci across the Old World monkeys are reasonably consistent. There appears to be more variability across species in the New World monkeys, with the night monkey showing the most simplistic morphology and the woolly monkey the most complex. The latter's parietal sulcal pattern in particular is quite extensive. Interestingly, the woolly monkey possesses the largest brain of the New World monkeys, potentially speaking to debates of whether gyrification is mostly driven by brain size differences (Mota and Herculano-Houzel 2015; Amiez et al. 2021).

\section{Left hemisphere}
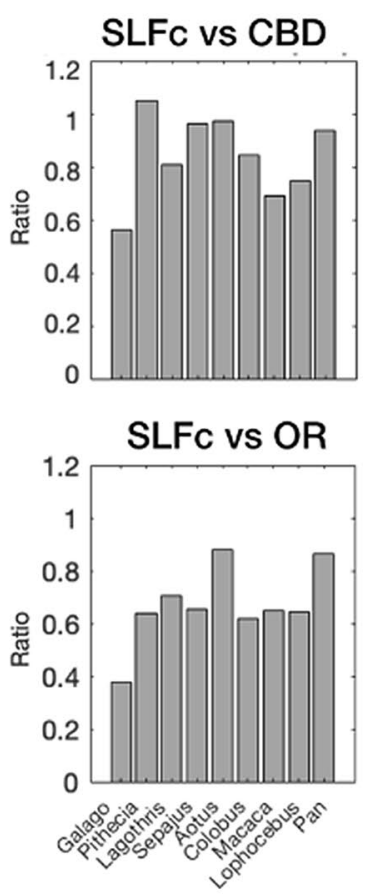

Right hemisphere

SLFc vs CBD
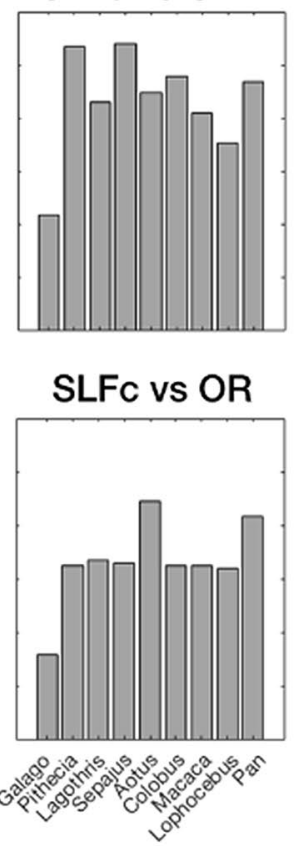

Left hemisphere
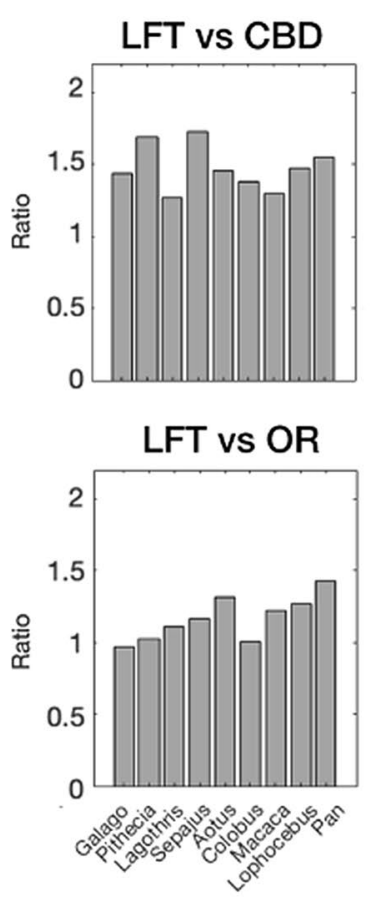

Right hemisphere

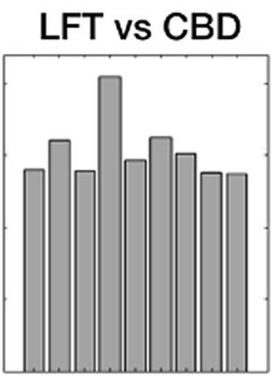

\section{LFT vs OR}

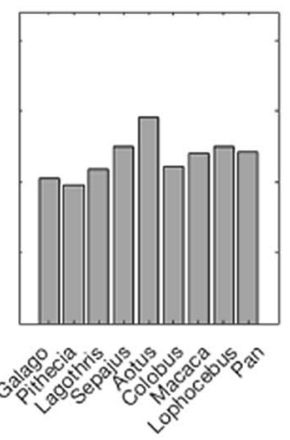

Fig. 5 Relative size of the SLF complex (SLFc) and longitudinal frontotemporal tract (LFT) in all studied species. Relative sizes of SLFc and LFT are displayed with respect to the dorsal cingulum bundle (CBD) and optic radiation (OR) for both hemispheres 
Given the limited information available for most of the species studied here, the proposed sulcal labels at this stage are not sufficient to prove that the sulci are the same across species. Indeed, whether sulcal patterning across species can be used for meaningful comparison is still a matter of debate (Amiez et al. 2019; Heuer et al. 2019), although relationships between sulcal anatomy and functional localization across individuals seem quite robust (Amiez et al. 2006; Eichert et al. 2021). Building on such results, recent work has used similarity in sulcal morphology to directly compare brains (Auzias et al. 2013) and show how it can be used to compare brains on the assumption that the sulci are directly comparable across species (Coulon et al. 2018). However, whether this assumption is justified needs additional testing. For instance, if one could establish similarities of cortical territories across multiple modalities, such as for instance their location in relation to prominent sulci, connectivity fingerprints, and cytoarchitecture, one can gain greater confidence in suggesting similarity of a given part of the brain across species (Mars et al. 2021).

Connectivity has been used to compare species quite extensively and the diffusion MRI data presented here can be used in this endeavour. This can be done by creating summary measures that describe, for instance, the efficiency of brains' wiring diagrams (Assaf et al. 2020) or by comparing the network organization of different brains (van den Heuvel et al. 2016, 2019). Additionally, one can use these data to reconstruct specific white matter tracts that, in turn, can be used to compare brain organization across species by creating whole brain white matter tract connectivity blueprints (Mars et al. 2018). We have here created four of such tracts in first instance, using recipes that are maximally similar across species and can therefore be meaningfully compared.

The cingulum bundle is a limbic tract carrying fibers from the medial temporal lobe, curving around the splenium of the corpus callosum, running along the white matter of the cingulate gyrus and terminating in the frontal, including subgenual, cortex (Catani et al. 2013). Recent tract tracing work in the macaque showed that many of the projections running through this fiber system only travel through part of the tract, leading to a definition of three subdivisions (Heilbronner and Haber 2014). We here reconstructed the dorsal subdivision that runs through the cingulate gyrus white matter along the corpus callosum. This tract is expected to scale with the size of the brain, as limbic fibers are generally thought to be quite conserved across primates (Folloni et al. 2019). Similarly, the optic radiation is thought to be quite conserved across different primates (Catani et al. 2003), making it a suitable reference tract.

We reconstructed two association fibers. We were able to reconstruct longitudinal parietal-frontal tracts in all species. The SLF is generally divided into three distinct bundles (Petrides and Pandya 1984), which in turn are separate from the arcuate fascicle that projects more ventrally (Petrides and Pandya 1988). However, as a first approximation and to establish our ability to reconstruct the SLFs, we here grouped them together in an 'SLF complex'. Comparison of this complex with the cingulum bundle and optic radiation shows that it is least extensive in the prosimian galago compare to other, anthropoid, primates. This is consistent with the so-called 'anthropoid elaboration' in cortico-cortical connectivity between parts of association cortex (Krubitzer 2009). Importantly, however, the SLF complex was present in the galago, consistent with tracer studies showing qualitatively similar parietal-frontal organization in galagos compared to other primates (Stepniewska et al. 2009; Kaas et al. 2011). Whether the anthropoid elaboration is a feature that can be predicted based on the size of the brain or whether if reflects a grade change between prosimian and simian primates is a matter that will require analysis in a full phylogenetic framework to address.

The presence of an inferior fronto-occipital tract (IFOF) or extreme capsule fiber complex has been accepted in the human brain for some time (Makris and Pandya 2008; Forkel et al. 2014), but its existence in the monkey has been controversial, due to the differential course of the tract suggested by tract tracing studies and some negative results using tractography in the macaque (Schmahmann and Pandya 2006; Takemura et al. 2017). Other authors, however, suggested a tract similar to the human IFOF could be identified in the macaque brain (Mars et al. 2016) when using similar tractography protocols in humans and macaques. More recently, dissection studies have confirmed the existence of a large white matter bundle along the course of the IFOF in both macaque and vervet monkeys (Decramer et al. 2018; Sarubbo et al. 2019). Here, we show the existence of an IFOF-like tract in all studied samples, including a frontal-occipital tract running medially in the temporal white matter sheet of the prosimian galago. Interestingly, a recent large-scale tractography study that included animals outside the primate order showed the presence of a ventral longitudinal tract across the mammalian order, although the lack of homologous temporal cortex in non-primate species means it is as yet unclear whether these tracts are homologous (Assaf et al. 2020).

The current results should of course be treated with caution. Although all animals were adults and the samples were selected for their high quality, the study is limited by its sampling range. More samples per species will allow one to address questions about within-species interindividual variability. A difficulty in increasing the sample number is the quality of the preserved brains required for this analysis. Brains should not be frozen and preserved with $24 \mathrm{~h}$ of death (D'Arceuil and de Crespigny 2007). Post-mortem cuts often made during brain extraction are making this endeavour more difficult as superficial cuts 
could impact on structural brain analysis (e.g., deformation-based morphometry) and deep cuts will interfere with the tractography analysis.

As indicated above, a full analysis of these data would ideally be done within in a formal phylogenetic framework (Harvey and Pagel 1991; Barton and Venditti 2013). Such analyses require quite extensive datasets. The current dataset adds to the growing number of initiatives that make data from a variety of species openly available to the scientific community (Thiebaut De Schotten et al. 2019). Such initiatives include the Primate Data Exchange (Milham et al. (2018); fcon_1000.projects.nitrc.org/indi/indiPRIME. html); the National Chimpanzee Brain Resource (www. chimpanzeebrain.org), the Japan Monkey Centre Primates Brain Imaging Repository (Sakai et al. (2018); j-monkey. jp/BIR/about_e.html); resources focused specifically on the marmoset (e.g., Liu et al. (2021), marmosetbrainmapping. org/data.html; Woodward et al. (2018), https://www.brain minds.riken.jp/atlas-package-download-main-page); and the brains provided in Heuer et al. (2019) and Navarrete et al. (2018). The latter of these contains structural images from the Primate Brain Bank, the same sample source as used in the present manuscript. In a parallel effort, resources for analysing data from non-human primate are also increasingly shared openly and support fora are emerging, including the Primate Resource Exchange (Messinger et al. 2021). These resources provide great opportunities for comparative science, and have already inspired and enabled projects of larger scale than possible by any one laboratory on its own.

Author contributions Data acquisition: DJA, LS, LR, AAK, NRS, BCT, WW, KLM, RBM; data analysis: DJA, KLB, JS, RBM, writing (first draft): RBM, writing (editing and approval final draft): all authors, project supervision: $\mathrm{MvdH}, \mathrm{RBM}$.

Funding The work of R.B.M. is supported by the Biotechnology and Biological Sciences Research Council (BBSRC) UK [BB/N019814/1] and the Netherlands Organization for Scientific Research NWO [45213-015]. LR is supported by funding from the Biotechnology and Biological Sciences Research Council (BBSRC) UK [BB/M011224/1]. The Wellcome Centre for Integrative Neuroimaging is supported by core funding from the Wellcome Trust [203139/Z/16/Z]. The work of MPvdH is supported by the Netherlands Organization for Scientific Research NWO [VIDI-452-16-015] and [ALW-179]. We also thank Corbert van Eeden and the entire staff of the Primate Brain Bank.

Data availability The pipeline for processing the spin-echo data uses FSL (www.fmrib.ox.ac.uk/fsl) and is implemented in the MR Comparative Anatomy Toolbox (Mr Cat; www.neuroecologylab.org) as the 'phoenix' module. The surface reconstruction pipeline is available at https://github.com/ardesch/nhp-freesurfer. All data are made available for scientific purposes on the WIN Digital Brain Bank (https:// open.win.ox.ac.uk/DigitalBrainBank/) subject to a Material Transfer Agreement. Tractography and results are available as a Data Sharing Collection link for reviewers: (https://data.donders.ru.nl/collections/ di/dcc/DSC_2020.00034 408).

\section{Declarations}

\section{Conflict of interest None.}

Ethical approval This work is done on post-mortem tissue of animals that have died of causes unrelated to research and as such does not require a Home Office license under the Animals (Scientific Procedures) Act 1986. All researchers were unaware of the existence and status of the animals before the samples arrived at the brain bank.

Open Access This article is licensed under a Creative Commons Attribution 4.0 International License, which permits use, sharing, adaptation, distribution and reproduction in any medium or format, as long as you give appropriate credit to the original author(s) and the source, provide a link to the Creative Commons licence, and indicate if changes were made. The images or other third party material in this article are included in the article's Creative Commons licence, unless indicated otherwise in a credit line to the material. If material is not included in the article's Creative Commons licence and your intended use is not permitted by statutory regulation or exceeds the permitted use, you will need to obtain permission directly from the copyright holder. To view a copy of this licence, visit http://creativecommons.org/licenses/by/4.0/.

\section{References}

Amiez C, Kostopoulos P, Champod A, Petrides M (2006) Local morphology predicts functional organization of the dorsal premotor region in the human brain. J Neurosci 26:2724-2731

Amiez C, Sallet J, Hopkins WD, Meguerditchian A, Hadj-Bouziane F, Ben Hamed S, Wilson CRE, Procyk E, Petrides M (2019) Sulcal organization in the medial frontal cortex provides insights into primate brain evolution. Nat Commun 10:1-14

Amiez C, Sallet J, Novek J, Hadj-Bouziane F, Giacometti C, Andersson J, Hopkins WD, Petrides M (2021) Chimpanzee histology and functional brain imaging show that the paracingulate sulcus is not human-specific. Commun Biol 4:54

Ardesch DJ, Scholtens LH, Li L, Preuss TM, Rilling JK, van den Heuvel MP (2019) Evolutionary expansion of connectivity between multimodal association areas in the human brain compared with chimpanzees. Proc Natl Acad Sci USA 116:7101-7106

Assaf Y, Bouznach A, Zomet O, Marom A, Yovel Y (2020) Conservation of brain connectivity and wiring across the mammalian class. Nat Neurosci 23:805-808

Auzias G, Lefèvre J, Le Troter A, Fisher C, Perrot M, Régis J, Coulon O (2013) Model-driven harmonic parameterization of the cortical surface: HIP-HOP. IEEE Trans Med Imaging 32:873-887

Avants BB, Tustison NJ, Song G, Cook PA, Klein A, Gee JC (2011) A reproducible evaluation of ANTs similarity metric performance in brain image registration. Neuroimage 54:2033-2044

Barton RA, Harvey PH (2000) Mosaic evolution of brain structure in mammals. Nature 405:1055-1058

Barton RA, Venditti C (2013) Human frontal lobes are not relatively large. Proc Natl Acad Sci USA 110:9001-9006

Behrens TEJ, Berg HJ, Jbabdi S, Rushworth MFS, Woolrich MW (2007) Probabilistic diffusion tractography with multiple fibre orientations: what can we gain? Neuroimage 34:144-155

Bryant KL, Glasser MF, Li L, Jae-Cheol Bae J, Jacquez NJ, Alarcón L, Fields A, Preuss TM (2019) Organization of extrastriate and temporal cortex in chimpanzees compared to humans and macaques. Cortex 118:223-243

Bryant KL, Li L, Eichert N, Mars RB (2020) A comprehensive atlas of white matter tracts in the chimpanzee. PLoS Biol 18:e3000971 
Buxton RB (1993) The diffusion sensitivity of fast steady-state free precession imaging. Magn Reson Med 29:235-243

Catani M, Jones DK, Donato R, Ffytche DH (2003) Occipito-temporal connections in the human brain. Brain 126:2093-2107

Catani M, Dell'Acqua F, Thiebaut de Schotten M (2013) A revised limbic system model for memory, emotion and behaviour. Neurosci Biobehav Rev 37:1724-1737

Chaplin TA, Yu H-H, Soares JGM, Gattass R, Rosa MGP (2013) A conserved pattern of differential expansion of cortical areas in simian primates. J Neurosci 33:15120-15125

Connolly CJ (1950) External morphology of the primate brain. Springfield: Thomas

Coulon O, Auzias G, Lemercier P, Hopkins WD (2018) Nested cortical organization models for human and non-human primate inter-species comparisons. Presented at the annual meeting of the organization for human brain mapping

D'Arceuil H, de Crespigny A (2007) The effects of brain tissue decomposition on diffusion tensor imaging and tractography. Neuroimage 36:64-68

Decramer T, Swinnen S, van Loon J, Janssen P, Theys T (2018) White matter tract anatomy in the rhesus monkey: a fiber dissection study. Brain Struct Funct 223:3681-3688

Eichert N, Verhagen L, Folloni D, Jbabdi S, Khrapitchev AA, Sibson NR, Mantini D, Sallet J, Mars RB (2019) What is special about the human arcuate fasciculus? Lateralization, projections, and expansion. Cortex 118:107-115

Eichert N, Robinson EC, Bryant KL, Jbabdi S, Jenkinson M, Li L, Krug K, Watkins KE, Mars RB (2020) Cross-species cortical alignment identifies different types of anatomical reorganization in the primate temporal lobe. Elife. https://doi.org/10.7554/eLife. 53232

Eichert N, Watkins KE, Mars RB, Petrides M (2021) Morphological and functional variability in central and subcentral motor cortex of the human brain. Brain Struct Funct 226:263-279

Falk D, Zollikofer CPE, Ponce de León M, Semendeferi K, Alatorre Warren JL, Hopkins WD (2018) Identification of in vivo Sulci on the external surface of eight adult chimpanzee brains: implications for interpreting early hominin endocasts. Brain Behav Evol 91:45-58

Fischl B (2012) Free surfer. Neuroimage 62:774-781

Folloni D, Sallet J, Khrapitchev AA, Sibson N, Verhagen L, Mars RB (2019) Dichotomous organization of amygdala/temporal-prefrontal bundles in both humans and monkeys. Elife. https://doi.org/10. 7554/eLife.47175

Forkel SJ, Thiebaut de Schotten M, Kawadler JM, Dell'Acqua F, Danek A, Catani M (2014) The anatomy of fronto-occipital connections from early blunt dissections to contemporary tractography. Cortex 56:73-84

Harvey P, Pagel M (1991) The comparative method in evolutionary biology. Oxford University Press, Oxford

Hecht EE, Gutman DA, Bradley BA, Preuss TM, Stout D (2015) Virtual dissection and comparative connectivity of the superior longitudinal fasciculus in chimpanzees and humans. Neuroimage 108:124-137

Hedges SB, Marin J, Suleski M, Paymer M, Kumar S (2015) Tree of life reveals clock-like speciation and diversification. Mol Biol Evol 32:835-845

Heilbronner SR, Haber SN (2014) Frontal cortical and subcortical projections provide a basis for segmenting the cingulum bundle: implications for neuroimaging and psychiatric disorders. J Neurosci 34:10041-10054

Hernandez-Fernandez M, Reguly I, Jbabdi S, Giles M, Smith S, Sotiropoulos SN (2019) Using GPUs to accelerate computational diffusion MRI: from microstructure estimation to tractography and connectomes. Neuroimage 188:598-615
Heuer K, Gulban OF, Bazin P-L, Osoianu A, Valabregue R, Santin M, Herbin M, Toro R (2019) Evolution of neocortical folding: a phylogenetic comparative analysis of MRI from 34 primate species. Cortex 118:275-291

Jenkinson M, Beckmann CF, Behrens TEJ, Woolrich MW, Smith SM (2012) FSL. Neuroimage 62:782-790

Kaas JH, van Eden CG (2011) Brain banks provide a valuable resource for comparative studies. Brain Behav Evol 77:65-66

Kaas JH, Gharbawie O, Stepniewska I (2011) The organization and evolution of dorsal stream multisensory motor pathways in primates. Front Neuroanat 5:34

Kellner E, Dhital B, Kiselev VG, Reisert M (2016) Gibbs-ringing artifact removal based on local subvoxel-shifts. Magn Reson Med 76:1574-1581

Krubitzer L (2009) In search of a unifying theory of complex brain evolution. Annu Rev NY Acad Sci 1156:44-67

Krubitzer L, Kaas J (2005) The evolution of the neocortex in mammals: how is phenotypic diversity generated? Curr Opin Neurobiol $15: 444-453$

Li L, Hu X, Preuss TM, Glasser MF, Damen FW, Qiu Y, Rilling J (2013) Mapping putative hubs in human, chimpanzee and rhesus macaque connectomes via diffusion tractography. Neuroimage 80:462-474

Liu C, Yen CC-C, Szczupak D, Tian X, Glen D, Silva AC (2021) Marmoset brain mapping V3: population multi-modal standard volumetric and surface-based templates. Neuroimage 226:117620

Makris N, Pandya DN (2008) The extreme capsule in humans and rethinking of the language circuitry. Brain Struct Funct 213:343

Marcus D, Harwell J, Olsen T, Hodge M, Glasser M, Prior F, Jenkinson M, Laumann T, Curtiss S, Van Essen D (2011) Informatics and data mining tools and strategies for the human connectome project. Front Neuroinform 5:4

Mars RB, Neubert F-X, Verhagen L, Sallet J, Miller KL, Dunbar RIM, Barton RA (2014) Primate comparative neuroscience using magnetic resonance imaging: promises and challenges. Front Neurosci 8:298

Mars R, Foxley S, Verhagen L, Jbabdi S, Sallet J, Noonan MP, Neubert F-X, Andersson JL, Croxson PL, Dunbar RIM, Khrapitchey AA, Sibson NR, Miller KL, Rushworth MFS (2016) The extreme capsule fiber complex in humans and macaque monkeys: a comparative diffusion MRI tractography study. Brain Struct Funct 221:4059-4071

Mars RB, Passingham RE, Neubert F-X, Verhagen L, Sallet J (2017) Evolutionary specializations of human association cortex. In: Kaas JH (ed) Evolution of nervous systems, 2nd edn. Academic Press, Oxford, pp 185-205

Mars RB, Sotiropoulos SN, Passingham RE, Sallet J, Verhagen L, Khrapitchev AA, Sibson N, Jbabdi S (2018) Whole brain comparative anatomy using connectivity blueprints. Elife. https://doi. org/10.7554/eLife.35237

Mars RB, O’Muircheartaigh J, Folloni D, Li L, Glasser MF, Jbabdi S, Bryant KL (2019) Concurrent analysis of white matter bundles and grey matter networks in the chimpanzee. Brain Struct Funct 224:1021-1033

Mars R, Jbabdi S, Rushworth M (2021) A common space approach to comparative neuroscience. Annu Rev Neurosci 44:69-86

McKavanagh R, Torso M, Jenkinson M, Kolasinski J, Stagg CJ, Esiri MM, McNab JA, Johansen-Berg H, Miller KL, Chance SA (2019) Relating diffusion tensor imaging measurements to microstructural quantities in the cerebral cortex in multiple sclerosis. Hum Brain Mapp 40:4417-4431

McNab JA, Jbabdi S, Deoni SCL, Douaud G, Behrens TEJ, Miller KL (2009) High resolution diffusion-weighted imaging in fixed human brain using diffusion-weighted steady state free precession. Neuroimage 46:775-785 
Messinger A, Sirmpilatze N, Heuer K, Loh KK, Mars RB, Sein J, Xu T, Glen D, Jung B, Seidlitz J, Taylor P, Toro R, Garza-Villarreal EA, Sponheim C, Wang X, Benn RA, Cagna B, Dadarwal R, Evrard HC, Garcia-Saldivar P, Giavasis S, Hartig R, Lepage C, Liu C, Majka P, Merchant H, Milham MP, Rosa MGP, Tasserie J, Uhrig L, Margulies DS, Klink PC (2021) A collaborative resource platform for non-human primate neuroimaging. Neuroimage 226:117519

Milham MP, Ai L, Koo B, Xu T, Amiez C, Balezeau F, Baxter MG, Blezer ELA, Brochier T, Chen A, Croxson PL, Damatac CG, Dehaene S, Everling S, Fair DA, Fleysher L, Freiwald W, Froudist-Walsh S, Griffiths TD, Guedj C, Hadj-Bouziane F, Ben Hamed S, Harel N, Hiba B, Jarraya B, Jung B, Kastner S, Klink PC, Kwok SC, Laland KN, Leopold DA, Lindenfors P, Mars RB, Menon RS, Messinger A, Meunier M, Mok K, Morrison JH, Nacef J, Nagy J, Rios MO, Petkov CI, Pinsk M, Poirier C, Procyk E, Rajimehr R, Reader SM, Roelfsema PR, Rudko DA, Rushworth MFS, Russ BE, Sallet J, Schmid MC, Schwiedrzik CM, Seidlitz J, Sein J, Shmuel A, Sullivan EL, Ungerleider L, Thiele A, Todorov OS, Tsao D, Wang Z, Wilson CRE, Yacoub E, Ye FQ, Zarco W, Zhou Y, Margulies DS, Schroeder CE (2018) An open resource for nonhuman primate imaging. Neuron 100:61-74.e2

Miller IF, Barton RA, Nunn CL (2019) Quantitative uniqueness of human brain evolution revealed through phylogenetic comparative analysis. Elife. https://doi.org/10.7554/eLife.41250

Mota B, Herculano-Houzel S (2015) Brain structure. Cortical folding scales universally with surface area and thickness, not number of neurons. Science 349:74-77

Navarrete AF, Blezer ELA, Pagnotta M, de Viet ESM, Todorov OS, Lindenfors P, Laland KN, Reader SM (2018) Primate brain anatomy: new volumetric MRI measurements for neuroanatomical studies. BBE 91:109-117

Passingham RE, Smaers JB (2014) Is the prefrontal cortex especially enlarged in the human brain? Allometric relations and remapping factors. Brain Behav Evol 84:156-166

Petrides M, Pandya DN (1984) Projections to the frontal cortex from the posterior parietal region in the rhesus monkey. J Comp Neurol 228:105-116

Petrides M, Pandya DN (1988) Association fiber pathways to the frontal cortex from the superior temporal region in the rhesus monkey. J Comp Neurol 273:52-66

Rilling JK, Glasser MF, Preuss TM, Ma X, Zhao T, Hu X, Behrens TEJ (2008) The evolution of the arcuate fasciculus revealed with comparative DTI. Nat Neurosci 11:426-428

Roumazeilles L, Eichert N, Bryant KL, Folloni D, Sallet J, Vijayakumar S, Foxley S, Tendler BC, Jbabdi S, Reveley C, Verhagen L, Dershowitz LB, Guthrie M, Flach E, Miller KL, Mars RB (2020) Longitudinal connections and the organization of the temporal cortex in macaques, great apes, and humans. PLoS Biol 18:e3000810

Sakai T, Hata J, Ohta H, Shintaku Y, Kimura N, Ogawa Y, Sogabe K, Mori S, Okano HJ, Hamada Y, Shibata S, Okano H, Oishi K (2018) The Japan monkey centre primates brain imaging repository for comparative neuroscience: an archive of digital records including records for endangered species. Primates 59:553-570

Sarubbo S, Petit L, De Benedictis A, Chioffi F, Ptito M, Dyrby TB (2019) Uncovering the inferior fronto-occipital fascicle and its topological organization in non-human primates: the missing connection for language evolution. Brain Struct Funct 224:1553-1567

Schmahmann JD, Pandya DN (2006) Fiber pathways of the brain. Oxford University Press, Oxford

Seidlitz J, Sponheim C, Glen D, Ye FQ, Saleem KS, Leopold DA, Ungerleider L, Messinger A (2018) A population MRI brain template and analysis tools for the macaque. Neuroimage 170:121-131

Smaers JB, Soligo C (2013) Brain reorganization, not relative brain size, primarily characterizes anthropoid brain evolution. Proc R Soc B 280:20130269

Smaers JB, Vanier DR (2019) Brain size expansion in primates and humans is explained by a selective modular expansion of the cortico-cerebellar system. Cortex 118:292-305

Stepniewska I, Cerkevich CM, Fang P-CY, Kaas JH (2009) Organization of the posterior parietal cortex in galagos: II. Ipsilateral cortical connections of physiologically identified zones within anterior sensorimotor region. J Comp Neurol 517:783-807

Takemura H, Pestilli F, Weiner KS, Keliris GA, Landi SM, Sliwa J, Ye FQ, Barnett MA, Leopold DA, Freiwald WA, Logothetis NK, Wandell BA (2017) Occipital white matter tracts in human and macaque. Cereb Cortex 27:3346-3359

Thiebaut de Schotten M, Dell'Acqua F, Forkel SJ, Simmons A, Vergani F, Murphy DGM, Catani M (2011) A lateralized brain network for visuospatial attention. Nat Neurosci 14:1245-1246

Thiebaut de Schotten M, Dell'Acqua F, Valabregue R, Catani M (2012) Monkey to human comparative anatomy of the frontal lobe association tracts. Cortex 48:82-96

Thiebaut De Schotten M, Croxson PL, Mars RB (2019) Large-scale comparative neuroimaging: Where are we and what do we need? Cortex 118:188-202

van den Heuvel MP, Bullmore ET, Sporns O (2016) Comparative connectomics. Trends Cogn Sci 20:345-361

van den Heuvel MP, Scholtens LH, de Lange SC, Pijnenburg R, Cahn W, van Haren NEM, Sommer IE, Bozzali M, Koch K, Boks MP, Repple J, Pievani M, Li L, Preuss TM, Rilling JK (2019) Evolutionary modifications in human brain connectivity associated with schizophrenia. Brain 142:3991-4002

Van Essen DC, Glasser MF, Dierker DL, Harwell J (2012) Cortical parcellations of the macaque monkey analyzed on surface-based atlases. Cereb Cortex 22:2227-2240

Warrington S, Bryant KL, Khrapitchev AA, Sallet J, Charquero-Ballester M, Douaud G, Jbabdi S, Mars RB, Sotiropoulos SN (2020) XTRACT - standardised protocols for automated tractography in the human and macaque brain. Neuroimage 217:116923

Woodward A, Hashikawa T, Maeda M, Kaneko T, Hikishima K, Iriki A, Okano H, Yamaguchi Y (2018) The brain/MINDS 3D digital marmoset brain atlas. Sci Data 5:180009

Yarnykh VL (2007) Actual flip-angle imaging in the pulsed steady state: a method for rapid three-dimensional mapping of the transmitted radiofrequency field. Magn Reson Med 57:192-200

Publisher's Note Springer Nature remains neutral with regard to jurisdictional claims in published maps and institutional affiliations. 